IJCOM 2021 November;1(2):84-8

\title{
Chronic Lymphocytic Leukemia in Farmer and Vegetable Seller
}

\author{
Firly Ratsmita ${ }^{*}$, Dewi S.Soemarko ${ }^{2}$ \\ ${ }^{1}$ Occupational Medicine Specialist Program, Faculty of Medicine, Universitas Indonesia, Jakarta, Indonesia \\ ${ }^{2}$ Occupational Medicine Division, Departement of Community Medicine, Faculty of Medicine, Universitas Indonesia \\ ${ }^{*}$ Corresponding address: Firly Ratsmita \\ Email: firlyratsmita@gmail.com
}

\begin{abstract}
Background: One of the risk factors for Chronic Lymphocytic Leukemia (CLL) is chemical exposure at work. The aim is to determine the relationship between patient who work as a farmer and vegetable seller with the incidence of CLL.

Case presentation: A-69-year-old man who has been working as a vegetable seller since 15 years and a chili farmer since 4 years ago. The patient was diagnosed with CLL. In his work as a vegetable seller and chili farmer, the patient gets five hazards ranging from physical, chemical, biological, ergonomic and psychosocial hazards so it is necessary to analyze whether there is a relationship between the patient's work and the current CLL disease.

Discussion: There are seven steps needed in the enforcement of occupational diseases. In addition, additional examinations are needed to determine the biomarkers of pesticides that can cause disease. This requires control for farmers and vegetable sellers who may be exposed to pesticides found in vegetables and chilies.

Conclusion: Vegetable seller and farmer have potential hazards, especially chemicals contained in pesticides which have a risk relationship with the incidence of CLL. However, to cause CLL, sufficient doses are required which are influenced by occupation, intensity of use and the type of specific chemical without the need for a long duration of exposure.

Keyword: chronic lymphocytic leukemia, farmer, vegetable seller, pesticide.
\end{abstract}




\section{Background}

In chronic lymphocytic leukemia there are the presence of (Cluster of Differentiation) $\mathrm{CD}^{+}, \mathrm{CD} 19^{+}, \mathrm{CD} 23^{+}$ which accumulates in the body that are classified as B-cell lymphoid neoplasms. The common mortality rate in patients with Chronic Lymphocytic Leukemia (CLL) is 2 to 3 years after diagnosis, but many patients survive 5 to 10 years. ${ }^{1-3}$

The incidence of new cases of CLL in the United States is around $1.2 \%$ with a mortality rate of about $0.7 \%$ of all cancer deaths. CLL experienced at the age of 70 years with an age range of 65-74 years. This occurrence in the elderly is thought to be a form of accumulation of toxins from chronic exposure. Incidence in men is up to 2 times more than women. ${ }^{1,4}$

In general, there are several risk factors for CLL including family history of cancer, radiation, pesticides, smoking, living in an agricultural environment exposed to herbicides and pesticides, reduced sun exposure, medical history of atopy, exposure to hepatitis $\mathrm{C}$ virus, and infection. There is an increased risk in industry and occupational exposures, especially to pesticides, agricultural agents which can lead to mechanisms of leukemogenesis role in the pathogenesis of CLL. Exposure to pesticides especially deltamethrin and herbicides is associated with CLL. ${ }^{4,5}$

CLL increases with a family history of leukemia with the presence of a polymorphic genetic locus found where there is active transcription in CLL cells and contain genes in controlling B cell development and signaling or immune function. The absence of an increase in cases in Japan after World War II shows the unclear link that ionizing radiation causes CLL. Increased incidence in individuals exposed to the breakdown of the Grenoble nuclear power plant. ${ }^{4,5}$

In studies conducted on exposure to insecticides (endosulfan, methomyl, pyrethrin, and chlorpyrifos), herbicides $(2,4 \mathrm{D}$, atrazine, diquat, and diuron), and fungicides (captan and thiram) on the incidence of CLL. The presence of exposure creates a cumulative exposure score (CES) on the use of pesticides and individual active substances and pyrethrin insecticides above 0,97 . The lowest correlation was monitored between the insecticide methomyl. The duration of pesticide exposure has a positive correlation with the prevalence and the coefficient of exposure intensity correlation above 0,71 . Therefore, insecticide exposure is associated as a risk of CLL from the workplace to organic pesticides and the highest relationship is exposed to organophosphate insecticides. The results of this study are similar to a cohort study on farmers and pesticide use, related to terbufos (organophosphate insecticide) and DDT (organochlorine insecticide) with CLL. ${ }^{2}$

\section{Case}

A-69-year-old man came with complaints of weakness and paleness. The patient had been hospitalized for 3 times for blood transfusions. The patient was declared to have leukemia after a bone marrow examination that led to CLL and continued with a positive (Cluster of Differentiation) CD 20, CD 5, Kappa, CD 43, CD 23 marker that led to CLL.

During this time, the patient worked as a seller and chili farmer. In the morning, patients usually trade in privately owned shops selling vegetables, meat, fish and chicken. Meanwhile, in the afternoon, the patient usually gardens to plant chilies with his children. The patient farms in the complex garden around his house. The patient started farming since 2017 by planting chili seeds, sowing, watering and also providing fertilizer. In addition, the patient also gave pesticides and fungicides to deal with pests and fungi that could appear in chili plants. The patient admitted that the use of pesticides and fungi was erratic and did not use certain brands. Patients use fertilizer every 10-14 days.

While working as a vegetable seller and farmer, the patient has several potential hazards. Physical hazards include ultraviolet (UV) rays, noise, inorganic dust. In chemical hazards, both as vegetable seller and farmer who can be exposed to pesticides, insecticides and fungicides. Biological hazards obtained include bacteria, viruses and fungi, as well as the presence of worms, insects or snakes. In ergonomic hazards, there can be awkward positions, repetitive movements that cause high risk to the back, and moderate risk to both wrists, neck and feet based on the brief survey. In the psychosocial aspect, work stress can occur as a result of dealing with customers as seller, and harvests that are not optimal due to the dry season. 


\section{Discussion}

In determining the diagnosis of occupational diseases, proper analysis is needed in the form of seven steps for establishing an occupational diagnosis. In the first step, the diagnosis in these patients is CLL although the patient tends to be asymptomatic, which usually occurs in $25-50 \%$ of CLL patients. A blood count, a blood smear, and B lymphocyte immunophenotyping, which identifies a clonal B cell population containing the CD5 antigen and B cell markers, are used to make the diagnosis. The presence of B lymphocyte counts $>5000 /$ microliter in peripheral blood for less than 3 months is used to diagnose CLL. Leukemic cells found on blood smears are typically small, mature lymphocytes with narrow cytoplasmic boundaries and dense nuclei that lack a visible nucleolus and have partly fragmented chromatin. These cells may be found mixed with larger or atypical cells, split cells, or prolymphocytes, which may up to $55 \%$ of the blood lymphocytes. The discovery of prolymphocytes in excess of this percentage would support the diagnosis of B-cell prolymphocytic leukemia (B-cell PLL). Gumprecht nuclear shadows, or smudge cells, found as cell debris, are another characteristic morphological feature found in CLL. CLL cells will express CD5 T cell antigens and B cell surface antigens CD19, CD20, and CD 23. Establishing a clinical diagnosis in this case was based on the results of bone marrow biopsy and examination of biomarkers in the blood that led to CLL in the presence of positive CD 20, CD 5, Kappa, CD 43, CD 23 markers. ${ }^{6}$

The next step is to determine the potential hazard in this patient's work. There are several risk factors that can be associated with agricultural work from Pesticides and synthetic fertilizers, diesel exhaust, UV radiation, biological dust, and zoonotic viruses and bacteria are all examples of environmental risks. In general, patients have 5 potential hazards when working, namely physical, chemical, biological, ergonomic and psychosocial hazards. The potential hazard that has the opportunity to be a risk factor for the incidence of CLL in workers who work as vegetable traders and chili farmers is with chemicals, especially insecticides and or fungicides commonly used in vegetable and chili plants. Classification of insecticides include organophosphates, organochlorines, carbamates, and pyrethroids. ${ }^{7,8}$

Although the content of pesticides used is still unclear, it is difficult to provide an overview of the pathophysiology of CLL due to pesticides. However, several references indicate that pesticides have the potential to cause carcinogenic effects, including CLL. In general, pesticides consist of insecticides (organophosphates, organochlorines, carbamates, pytheroids, rotenoids), herbicides (phenoxy acetic acid, phenoxy benzoic acid, thiocarbamates, triazines, anilides, dipyridyl compounds, phosphonates), fungicides (thiophthalimides, thiocarbamates, ethylene bis dithiomates), fumigants and ethylene bisdithiocarbamate (EBDCs). The recommended use of pesticides is only for the eradication of pests found and is not recommended routinely, however there are some farmers using the wrong dose and mix including those carried out by patient because there is no special training in growing chilies. Inappropriate use can have an effect on the environment including causing human exposure both acutely and chronically. Most of the use of pesticides that are not in accordance with the target will pollute the air, water and soil environment which can affect the population in agricultural areas and people who consume food contains pesticide. The high risk of herbicide exposure is related to frequent exposure to insecticides. In addition, use in a closed room can be a risk factor compared to the outdoor. ${ }^{9,10}$

Several studies have shown the effect of pesticides on human health. Pesticides have neurotoxin effects that result in Alzheimer's and Parkinson's disease, and cause neuronal disorders and degenerative diseases, fetal effects, congenital anomalies and carcinogenic in humans. Fungicides and insecticides are also neurotoxins that have synapse modulating effects on neurotransmitters. Increased use of pesticides can increase the incidence of leukemia, clone thyroid, brain and several other types of cancer. Epidemiological studies show that many pesticides are carcinogenic such as sulfallates, organochlorines and sulfates, while other pesticides, lindane and chloradane, are the cause of tumors. ${ }^{7}$

In organophosphate studies in animals, there was a differential disorder of hematopoietic cells (parathion 4 $\mathrm{mg} / \mathrm{kg} \mathrm{po}$ ) which induces weak clastogenicity in bone marrow cells. In vitro exposure to organophosphates in bone marrow cells or CD 34+ can reduces progenitor capacity to differentiate and sever DNA doublestranded and mixed-lineage leukemia (MLL) gene rearrangements. Organophosphates cause DNA damage (isophenphos), hypermethylation or tumor suppressor genes (diazinon) or chromosomal damage 
(phenithrothion). ${ }^{11}$

Pesticides also cause leukemia in children with a history of pesticide exposure in the mother during pregnancy, especially the pesticide permethrin with a seven times risk of developing leukemia. Permethrin is commonly used to protect pets from flea production and to kill mosquitoes. These chemicals can also change the nervous system acting on insects, in some studies are also considered carcinogenic. Leukemia in children is caused by DNA changes during infancy. Based on research it was found that the time from pregnancy to 11 months of breastfeeding is very important for children and if there is a history of exposure to pesticides then they have twice the chance of developing leukemia in children. ${ }^{7}$

In this case, the patient was diagnosed with CLL at the age of 69 years, whereas CLL is common in the elderly. This suggests that there is a toxic accumulation of chronic exposure in lymphoid or other tissues that interacts with the work history of vegetable seller for 15 years and chili farmer for 4 years. Increased accumulation of biologically active metabolites especially in lymphoid can react irreversibly to DNA mutations that can induce chromosomal aberrations in CLL. This is thought to trigger mutagenesis and carcinogenesis. ${ }^{4}$

Organochlorines (e.g., aldrin, chlordane, dichlo rodiphenyltrichloroethane, dieldrin), lead, arsenate, creosote, and sulfallate), as well as lead arsenate, creosote, and sulfallate, have all been shown to cause cancer in animals. Several studies have shown an association of non-Hodgkin's lymphoma with phenocyacetic acid, organophosphates and organochlorines from the analysis of epidemiological studies. Leukemia is associated with the use of insecticides, carbamates, organochlorines, organophosphates, herbicides, and zoonotic viruses. In the study of Boros and William, they reported exposure to In dose-dependent leukemic cell proliferation, leukemic cell lines (K562) were exposed to increasing dosages of an organophosphate insecticide (isofenphos). ${ }^{?}$

To cause a carcinogenic effect, it is necessary to calculate pesticide exposure measurements including the duration of exposure or work as vegetable seller and farmers, increase in harvest time or duration, and use of specific chemicals (duration and frequency). This can be used as an assessment of the estimated exposure to workers. This calculation can show the duration and intensity of exposure that can be related to the time and disease experienced. In the exposure classification, there is a consideration of low exposure to chemicals, especially in the work of vegetable traders and medium or high exposure to the work of chili farmers in these patients. Questionnaires can help determine the amount of pesticide exposure, how to work as well as the wearing of personal protection equipment. Although in this case, the patient admitted that he did not use certain brands of pesticides or certain ingredients, exposure to pesticides could increase the risk of health problems due to the patient's inappropriate use of personal protective equipment. Patients do not use special personal protective equipment while working, including not using special clothes and washing hands or cleaning after work or contamination with pesticides.?

Pesticides have been linked to prostate cancer, nonHodgkin lymphoma, leukemia, multiple myeloma, bladder cancer, and colon cancer in a number of studies. The pesticide related to leukemia contains thiocarbamate and organophosphate with a relative risk of 2.36 (1.16$4.84)$ and 2.38 (1.35-4.21). In addition, the content of pesticides containing chlorinate phenol, triazine, pyrethroid is also associated with the risk of leukemia. This increases the risk of farmers to develop cancer $(\mathrm{OR}=1.459,95 \%$ CI 1.229-1.731) compared to nonfarmers. Apart from farmers, the common household use of pesticides is pyrethroid. This insecticide is associated with hematological cancer, especially leukemia. The most common exposure to the human body can be through the oral and skin. After entering the organism, pyrethroids undergo metabolism and elimination. In mammals, pyrethroids are rapidly metabolized and eliminated in the urine and faeces several days after exposure. Permethrin reaches peak concentrations hours after oral exposure in blood plasma and is eliminated within 48 hours in the urine. The pyrethroid metabolic pathway is via ester hydrolysis and oxidation, which is mediated by carbocyl esterase and cytochrome P450. Although pyrethroids are eliminated faster than other pesticides, there is a danger of chronic effects, namely the development of cancer. ${ }^{8,12}$

To develop into cancer, it is also necessary to have other environmental factors such as smoking, alcohol, and ultraviolet radiation that can induce cancer. The risk of cancer due to smoking, especially lung cancer occurs in heavy smokers. The patient was a moderate smoker based on the brinkman index category with a score of $300 .^{13}$

The development of cancer can occur due to DNA damage. The molecular effects of pyrethroids which begin by binding directly to DNA, causing DNA 
methylation and other epigenic modifications that will have an effect on changes in gene expression and disrupt estrogen and androgen receptors which ultimately cause DNA damage through mechanisms that produce quinones and semiquinone. Another mechanism is that the pyrethroids will cause oxidative stress which can lead to DNA damage. Pyrethroids function as agonists or antagonists of estrogen, androgen, and thyroid hormone receptors, causing endocrine disruption. In general, pesticides cause DNA damage, oxidative stress, metabolic output and related thyroid effects in humans. ${ }^{8,12}$

\section{Conclusion}

Establishing a work-related diagnosis requires further investigation and examination, especially for examination of biomarkers of pesticide content, including organophosphate, organoflorin or carbamates. One of the factors that determine the cause of CLL due to pesticides is the cumulative dose response without determining the duration of chronic exposure which can cause chronic effects including cancer development. For this reason, further occupational management is needed regarding the control that can be carried out and the determination of return to work in patients with CLL, both as vegetable sellers and farmers.

\section{Reference}

1. Nicholas C, Chen S-S, R. RK. Chronic lymphocytic leukemia [Internet]. Cold Spring Harbor Perspectives in Medicine. 2021. Available from: http://perspectivesinmedicine.cshlp. org/content/11/2/a035220.full.pdf+html

2. Benavente Y, Costas L, Rodríguez-Suarez MM, Alguacil J,
Santibáńez M, Vila J, et al. Occupational exposure to pesticides and chronic lymphocytic leukaemia in the MCC-Spain study. Int J Environ Res Public Health. 2020;17(14):1-11.

3. Muhammad A Mir. Chronic Lymphocytic Leukemia (CLL): Practice Essentials, Pathophysiology, Etiology [Internet]. Medscape. 2020. Available from: https://emedicine.medscape. com/article/199313-overview

4. Karakosta M, Delicha EM, Kouraklis G, Manola KN. Association of various risk factors with chronic lymphocytic leukemia and its cytogenetic characteristics. Arch Environ Occup Heal. 2016;71(6):317-29.

5. Hallek M, Shanafelt TD, Eichhorst B. Chronic lymphocytic leukaemia. Lancet [Internet]. 2018;391(10129):152437. Available from: http://dx.doi.org/10.1016/S01406736(18)30422-7

6. Hallek M. Chronic lymphocytic leukemia: 2017 update on diagnosis, risk stratification, and treatment. Am J Hematol. 2017;92(9):946-65.

7. U A, MF M. Pesticide Exposure and Human Health: A Review. J Ecosyst Ecography. 2016;01(s5).

8. Curl CL, Spivak M, Phinney R, Montrose L. Synthetic Pesticides and Health in Vulnerable Populations: Agricultural Workers. Curr Environ Heal reports. 2020;7(1):13-29.

9. Alavanja MCR, Hoppin JA, Kamel F. Health effects of chronic pesticide exposure: Cancer and neurotoxicity. Annu Rev Public Health. 2004;25:155-97.

10. Pluth TB, Zanini LAG, Battisti IDE. Pesticide exposure and cancer: an integrative literature review. Saúde em Debate. 2019;43(122):906-24.

11. 11. Van Maele-Fabry G, Gamet-Payrastre L, Lison D. Household exposure to pesticides and risk of leukemia in children and adolescents: Updated systematic review and meta-analysis. Int J Hyg Environ Health [Internet]. 2019;222(1):49-67. Available from: https://doi.org/10.1016/j. ijheh.2018.08.004

12. Navarrete-Meneses MDP, Pérez-Vera P. Pyrethroid pesticide exposure and hematological cancer: Epidemiological, biological and molecular evidence. Rev Environ Health. 2019;34(2):197-210.

13. Arumsari D, Artanti KD, Martini S, Widati S. the Description of Smoking Degree Based on Brinkman Index in Patients With Lung Cancer. J Berk Epidemiol. 2019;7(3):249. 